\title{
Fume Suppressant of Hot Mix Asphalt Mixture: A Review
}

\author{
Xinyi Zhou ${ }^{1, \mathrm{a}^{*}}$, Jiayao Liu ${ }^{1}$, Shibing $\mathrm{Xu}^{1}$ \\ ${ }^{1}$ School of Highway, Chang'an University, Middle Section of Nan, Erhuan Road, Xi' an 710064, China
}

\begin{abstract}
Asphalt is a black, thermoplastic, hydrocarbon material extracted from crude oil and widely used in road construction all over the world. In order to meet the requirements of construction technology, asphalt always needs to be heated to flow state in the process of mixing and paving, during which a large amount of asphalt fume is released. Asphalt fume is a complex mixtures of inorganic compound gases, volatile organic compounds and particulate matter, which poses a serious threat to the natural environment and the health of practitioners. In order to solve this problem, researchers have developed different types of fume-suppressant. In this literature review, the properties, mechanisms and effects of all kinds of commonly used fumesuppressants were discussed in detail. Finally, the problems existing in the development of asphalt fumesuppressant and corresponding improvement considerations were pointed out.
\end{abstract}

\section{Introduction}

Asphalt in the high temperature mixing and paving process will produce a lot of fume, which is harmful to the natural environment and the health of employees. The irritating smell of asphalt fume seriously affects the quality of life of nearby residents. At the same time, the performance of asphalt material itself will be deteriorated due to the emission of fume.

Most of the traditional asphalt fume treatment technologies need fixed equipment so that they are unsuitable for the construction site. In recent years, in order to achieve the goal of sustainable development of highway engineering, many scholars have conducted research on asphalt fume-suppressant. In this paper, the characteristics and mechanism of fume-suppressants are discussed in order to provide reference for the research and application of fume-suppressants in road engineering.

\section{The type and mechanism of fume suppressant}

Existing fume suppressants are often divided into the following categories: flame-retardant, adsorbent, polymer modifier and novel fume suppressant.

\subsection{Flame-retardant}

Asphalt flame-retardant is a modifier used to improve the oxygen concentration required for the combustion of asphalt materials, delay the spread of fire and reduce the flammability of asphalt[1]. Flame-retardants can be divided into halogen, inorganic and intumescent flameretardants. By eliminating the active free radicals which are conducive to flame propagation, the halogenated flame-retardants can inhibit the oxidation and decomposition of combustibles. However, in fact, the smoke production also increases[2], which is harmful to the environment and human health. Therefore it has been gradually replaced by other environmental friendly flameretardants. Most inorganic flame-retardants and intumescent flame-retardants can not only improve the flame retarding performance of asphalt, but also inhibit the volatilization of light components and isolate oxygen, so as to reduce the smoke emission of asphalt.

Metal hydroxide is one of the most widely used inorganic flame retarding fume-suppressant, and the common types include magnesium hydroxide, aluminum hydroxide and calcium hydroxide. When the matrix is heated, the inorganic flame-retardant can absorb heat and dehydrate in a short time, reducing the internal temperature of the matrix and preventing the decomposition of asphalt components [3,4]. At the same time, hydroxide can neutralize the acid substances produced by the decomposition of asphalt and reduce the fume. However, such smoke suppressant needs to be used in large quantities to be effective.

Intumescent flame-retardant can be divided into chemical intumescent flame-retardant and physical intumescent flame-retardant. Chemical intumescent flame-retardant is a kind of compound flame-retardant with nitrogen and phosphorus as the main components. It is generally composed of carbon source, acid source and gas source [5], which are respectively called carbonizing agent, dehydrating agent and foaming agent and the representative substances are pentaerythritol, ammonium polyphosphate and melamine. The commonly used physical intumescent flame-retardant is expanded graphite. Intumescent flame-retardant can form a compact carbon foam layer on the surface of asphalt, which can block heat transfer, isolate oxygen, inhibit decomposition of asphalt, and reduce fume production[6]. In view of the fact that the fume suppression mechanism of expanded graphite 
mainly lies in adsorption, it is classified into the adsorbent for subsequent discussion.

\subsection{Adsorbent}

With large porosity and surface area, adsorbent has strong adsorption capacity for light components of asphalt fume. According to the principle of adsorption, it can be divided into physical adsorption and chemical adsorption. Physical adsorption is characterized by no chemical reaction between the adsorbent and the adsorbate. The high free energy generated by the high surface area of the adsorbent makes the light components adhere to the surface and gradually diffuse to the inner pore. Finally, fume are fixed in the pore due to the intermolecular force[3]. Through the polar functional groups and characteristic structures of the surface, such as carboxyl, hydroxyl, benzene ring and so on, the chemical adsorbent reacts with asphalt fume to inhibit the escape of fume molecules in thermal motion[7]. The commonly used adsorbent fume-suppressant in the research mainly include activated carbon[8], expanded graphite[9] and coumarone resin, $\mathrm{C} 9$ petroleum resin, phthalocyanine blue and other adsorbents for polycyclic aromatic hydrocarbons.

The rich pore structure and large surface area of activated carbon make it have dual effects of physical adsorption and chemical adsorption. Xiao et al. analyzed the effect of activated carbon on the amount of VOCs with UV spectrophotometer, and the results confirmed that VOCs were significantly reduced after the introduction of activated carbon[8]. However, the preparation of activated carbon modified asphalt will generate a lot of irritating smoke, which will cause new pollution problems[10].

Expanded graphite is a kind of graphite intercalation compound which is treated by inorganic acid. The results show that the expanded graphite has affinity to asphalt, which will swell when mixed with hot asphalt, contributing to the diffusion of light components into graphite layer or adsorption on the surface. There is van der waals between the expanded graphite layer and asphalt components[9]. As shown in Figure 1, due to the expansion of graphite layer, the adsorption and crystallization nucleation of PAHs by expanded graphite can be further enhanced, which can significantly reduce the content of aromatic compounds and sulfur compounds in asphalt fume.

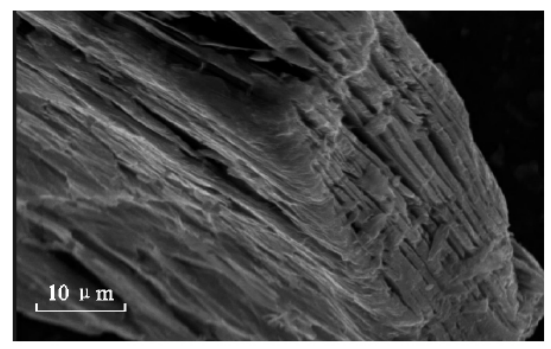

(a) original expanded graphite

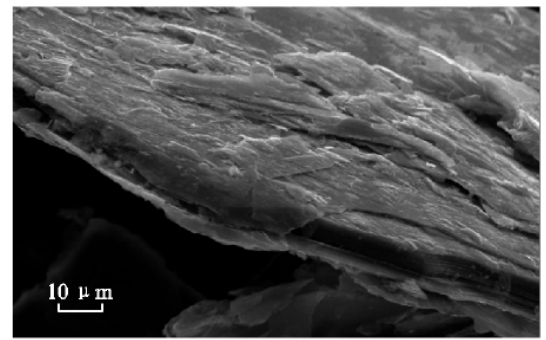

(b) expanded graphite eluted from solvent

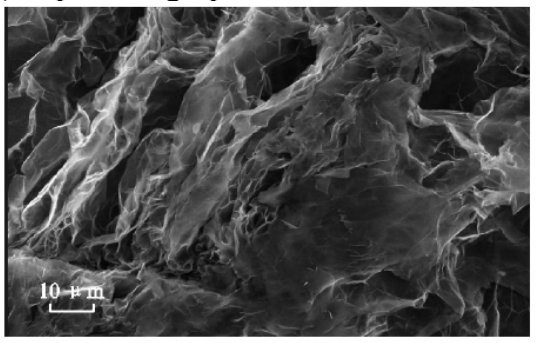

(c) expanded graphite eluted from hot asphalt Fig1. SEM images of expanded graphite ${ }^{|9|}$

The molecular structures of coumarone resin, C9 petroleum resin and phthalocyanine blue all contain benzene ring and substances with strong electron absorption effect. According to the principle of "similarity and intermiscibility" and molecular electrophilic substitution reaction, this kind of adsorbent fumesuppressant can react with PAHs in asphalt fume, effectively inhibiting the emission of polycyclic aromatic hydrocarbons[11].

In fact, the process of each adsorbent on asphalt fume is quite complex, so it is difficult to strictly divide a certain adsorbent into physical or chemical adsorbent. And usually both work at the same time to achieve ideal fume suppression effect.

\subsection{Polymer modifier}

Polymer modifier is one of the most commonly used modifiers for road asphalt. It can not only improve the road performance of asphalt mixture, but also has the function of fume suppression and environmental protection, such as SBS, SBR, rubber, PE. The mechanism of polymer fume suppressor is mainly physical crosslinking, chemical crosslinking and grid structure binding.

Taking the most widely used SBS modifier as an example, SBS will absorb the light components in the asphalt to swell, then disperse more evenly. Each SBS structure contains multiple functional groups, i.e. one double bond, two phenyl groups, and several methylene groups connected with double bond and benzene ring. It is easy to react with heteroatoms and active groups in asphalt to form SBS-asphalt grafts and network structure[12], decreasing the gibbs free energy and surface tension of the system, forming a stable whole of modified asphalt. The combination of asphalt and polymer not only increases the molecular cohesion and inhibits the decomposition of asphalt, but also makes the small asphalt molecules penetrate into the space network structure, which 
constrains the volatilization of light components and reduces the fume production[13].

\subsection{Novel fume suppressant}

\subsubsection{Nano fume suppressant}

Nanomaterials refer to materials with at least one dimension of nanometer scale $(1 \sim 100 \mathrm{~nm})$ in threedimensional space. The system composed of nano particles usually shows many special properties, such as surface and interface effect, small size effect, chemical activity and adsorption, making the nano materials excellent fume suppression performance. At present, the widely applied nano fume suppressants are layered nano silicate and nano calcium carbonate.

The fume suppression performance of nano fume suppressant is mainly related to the following characteristics. Firstly, the inner atomic spacing of nanoparticles is very small, the number of surface atoms accounts for a large proportion, and its thermal movement is much more intense than that of internal atoms. Second, due to the large surface area and insufficient surface atom coordination, nano materials have stronger adsorption than similar bulk materials. Finally, the surface layer of nano particles often contains bands. The type and size of electric functional groups affect the electrostatic interaction between particles, and further cause changes in macroscopic properties. In the process of mixing with asphalt, the nano fume suppressant will absorb part of the light components in the asphalt to form a stable group, so as to reduce the volatilization of the light components during the heating process, and cut down the generation of asphalt fume.

In addition to the universal characteristics of nano calcium carbonate, the special structure of layered silicate endows it better fume suppression performance. The frequently-used layered silicates cover montmorillonite, rectorite, vermiculite and kaolin[14]. The mixture of asphalt and layered silicate will form two types of structure: two-dimensional ordered intercalation structure or exfoliation structure, thus prolonging the average penetration path length of oxygen molecules and hindering their penetration in asphalt. This can effectively inhibit the thermal movement and the formation of oxygen-containing functional groups in asphalt molecules, reducing the loss of light components and the mass loss rate of asphalt. Yang et al. prepared aluminum hydroxideorganic montmorillonite composite modified asphalt, and measured the concentration of VOC in the mixture fume by VOC gas detector. The results showed that the aluminum hydroxide-organic montmorillonite composite material can effectively inhibit the fume emission[15].

Another attempt of nano fume suppressant is mesoporous silica hollow nano spheres. As shown in Figure 2, the inner hollow nanospheres with mesoporous channels on the surface have unique properties such as small density, large surface area, large porosity and strong loading capacity $[3,16]$, which has a broad application prospect in the field of adsorbent. Shu et al. innovatively applied it to asphalt fume suppression, and learned that the adsorption process can be roughly divided into three stages: VOC was adsorbed on the surface - VOC spreaded into the pores - VOC was fixed in the pores. The result declared that mesoporous silica hollow nanospheres can significantly reduce the VOC emission of asphalt, especially have a strong adsorption effect on hydrocarbon derivatives[3].
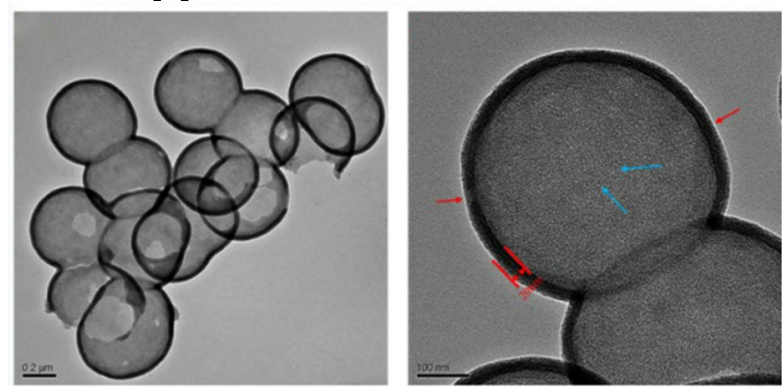

Fig2. TEM images of mesoporous silica hollow nano-spheres ${ }^{[3]}$

\subsubsection{Layered double hydroxides}

Layered double hydroxides (LDHs), also known as hydrotalcite like or layered composite metal hydroxides, is generally composed of hydroxides of two or more metal elements. There is a large number of hydroxyl, carbonate, crystal water and amorphous water between the layers. When heated, it can absorb energy to decompose and release $\mathrm{H}_{2} \mathrm{O}$ and $\mathrm{CO}_{2}$, and stop the generation of fume. The particular layered structure of LDHs makes it possible to be intercalated with various ions. As a low fume, nontoxic green flame-retardant, LDHs has been used in a variety of polymers. For the past few years, some scholars try to use LDHs to improve the fume suppression performance of asphalt. It is proved that LDHs modifier can reduce the mass loss rate[17], volatilization rate and VOC emission of asphalt, and has prominent inhibition effect on small molecule.

\subsubsection{Tourmaline}

Tourmaline is a kind of silicate mineral with piezoelectric, thermoelectric, permanent spontaneous polarization effect and spontaneous release of negative ions. In environmental science, tourmaline has the functions of dust suppression, adsorption of harmful gases, and removal of peculiar smell. Tourmaline can be applied to modified asphalt to improve its deformation resistance[18], flame retardant and fume suppression properties. The fume suppression performance of tourmaline is reflected in the following three aspects. Firstly, tourmaline powder has large surface area and high surface activity, which can facilitate the combination of unsaturated bond and free radical, thus reducing the concentration of free radical, and finally hindering the formation of asphalt fume. Secondly, the charge generated by the piezoelectric and thermoelectric properties of tourmaline has adsorption effect on asphalt fume. In addition, tourmaline contains a large proportion of hydrated alumina. In the heating process, crystal water will absorb heat and volatilize, thus achieving the effect of cooling and emission reduction. 


\section{Disscussion}

To sum up, some achievements have been made in the research of fume suppressant of hot mix asphalt mixture, but there are still some problems to be further studied in the future.

(1) To establish a unified test method and index for fume suppressant. At present, the test device and parameters selected by each researcher are different, such as heating temperature, heating time, asphalt content, stirring condition, making the test results less comparable. The existing indexes are basically directly determined emissions, lack of asphalt properties to characterize the essential impact of fume emissions. Besides, direct measurement is mainly VOC, ignoring $\mathrm{SO}_{2} 、 \mathrm{NO}_{x} 、 \mathrm{CO}$ and other inorganic compound gases.

(2) Speed up the formulation of asphalt emission limit and related specifications. At present, the research on asphalt fume at home and abroad is mainly qualitative. There is no evaluation standard for asphalt fume composition. The emission limit of asphalt fume during highway construction is not clear, so it is impossible to evaluate the risk of asphalt fume release under construction conditions.

(3) The application form of fume suppressant and its influence on pavement performance of asphalt mixture should be further explored. Most of the studies of fume suppressants lie in the laboratory and certain test section, and have not been widely used in engineering practice. Therefore the effect and application scope of all kinds of fume suppressants need to be further studied. Due to the diversity of asphalt sources and the complexity of asphalt composition structures, the influence of volatilization of asphalt fume on asphalt composition and properties is worthy of attention.

(4) To promote the research of novel fume suppressants and related technologies. Lately, although some new materials, such as layered silicate and tourmaline powder, have been introduced into the field of asphalt fume suppression, the mechanism has not been completely explicit and should be paid full attention to. Meanwhile, the effect is often influenced by the compatibility of fume suppression and asphalt. Therefore, on the basis of the existing research, it is also an urgent issue to develop new fume suppressant with better effect and wider application and its surface modification technology.

\section{Conclusions}

(1) When heated, asphalt will produce a large number of poisonous fume, greatly threatening the natural environment and practitioners' health. Fume suppressant can effectively restrain fume release, which has the good social benefits.

(2) According to the characteristics of fume suppressants, they can be divided into four types: flame retardant, adsorbent, polymer modifier and novel fume suppressant. The representative substances are metal hydroxide, activated carbon, SBS and hydrotalcite etc.

(3) At present, the study of fume suppressant has made some progress, but the application form, the applicable scope, and mechanism still need further research.

\section{References}

1. Tian Yu, Zheng Suining,Kuang Dongliang, Application and Research Progress of Asphalt Flame Retardant[J]. Petroleum Asphalt , 2015. 29(05): 6-10.

2. Ou Yuxiang, Chen Yu, Han Tingjie, et al. Development Ad- vances in Smoke Suppressants for Plastics and Their Smoke Suppression M lechanisms[J]. China Plastics, 2006(02): 6-12.

3. Shu Benan, Inhibition Effect and Mechanism of Mesopor- ous Silica Hollow Nano-Spheres on Asphalt VOCs[J]. 2019.

4. Xu Tao, Wang Hongchang,Huang Xiaoming, et al., Inhibitory action of flame retardant on the dynamic evolution of asphalt pyrolysis volatiles[J]. Fuel. 2013. 105.

5. Zhang Jinkai, Ma Li,Ge Weijuan, et al. Research Status of Intumescent Flame retarded Polypropylene[J]. Materials Reports, 2015. 29(05): 68-72.

6. Pan Liwen, Li Kongzhe,Xiao Yang, et al. Research Status of Intumescent Flame Retardant[J]. Materials Reports, 2016. 30(05): 27-32.

7. Peng Xuya, Li Zhiyang, Study On Regularity Of Fumes Emitting From Asphalt[J]. Intelligent Automation \& Soft Comuption, 2010. 16(5).

8. Xiao Yue, Wan Meinan,Jenkins Kim, et al., Using Activat- ed Carbon to reduce the volatile organic compounds from bituminous materials[J]. Journal of Materials in Civil Engi- neering, 2017. 29(10): 4017161-4017166.

9. Huang Gang, He Zhaoyi, Zhou Chao, et al. Suppression Mechanism of Expanded Graphite for Asphalt Fume and Dynamic Performance of Asphalt Mixture of Fume Supp- ression [J]. China Journal of Highway and Transport, 2015. 28(10): 1-10.

10. Yang Xiwu, Peng Xuya,Zhang Xingyu, Experiments on the asphalt fume suppression agents and properties of asphalt concrete with fume suppression agent [J]. Journal of Chong- qing University (Natural Science Edition), 2013. 36(12): 70-78.

11. Liu Jing, He Zhaoyi,Huang Gang, Development and Appli- cation of Fume Suppressant[J]. Petroleum Asphalt, 2014, 28(03) : 63-67.

12. Li Zhiyang,Xuya Peng, Suppression Analysis of Polymers on High Temperature Asphalt Fume[J]. Industrial Safety and Environmental Protection, 2015, 41(10): 24-26.

13. Liu DaLiang, Huang YunYong, Zheng JianLong, et al. Study of Mechanism of Flame-Retardant SBSModified Asphalt[J]. International Journal of Pavement Research and Technology, 2009, 2(4). 2009. 2: 162-165.

14. Zhang Henglong, Shi Caijun,Han Jun, et al., Effect of organic layered silicates on flame retardancy and 
aging properties of bitumen[J]. Construction \& Building Materials, 2013, 40(Mar.):1151-1155.

15. Yang Xiaolong, Shen Aiqin,Su Yuxuan, et al., Effects of alumina trihydrate (ATH) and organic montmorillonite (OMMT) on asphalt fume emission and flame retardancy properties of SBS-modified asphalt[J]. Construction and Building Materials, 2020. 236.

16. Wang Dong, Wang Jian, Hu Yuan. Low-cost synthesis of hollow mesoporous silica spheres and its application in the removal of aromatic volatiles[J]. Materials Letters, 2017. 208.

17. Cui Peiqiang, Zhang Honghua, Wu Shaopeng, Influence of High-Temperature Volatilization on Performance of Bitumi- nous Binder[J]. Key Engineering Materials, 2014, 599: 164-167.

18. Ye Qunshan, Dong Wenzhuo, Wang Shipei, et al. Research on the Rheological Characteristics and Aging Resistance of Asphalt Modified with tourmaline[J]. Materials (Basel, Switzerland), 2019,13(1). 